\title{
RELATIONSHIP OF AIRWAY DIMENSIONS WITH AIRFLOW LIMITATION OR LUNG VOLUMES IN CHRONIC OBSTRUCTIVE PULMONARY DISEASE (COPD)
}

\author{
Masaru Hasegawa, Yasuyuki Nasuhara, Yuya Onodera, Hironi Makita, Tomoko Betsuyaku, Masaharu Nishimura and \\ Hokkaido COPD Cohort Study Group \\ First Dept of Medicine, Hokkaido University School of Medicine, Sapporo, Japan
}

WINNING ABSTRACT: We have recently developed new software to obtain longitudinal images and accurate short axis images of airways with an inner diameter $>2 \mathrm{~mm}$ located anywhere in the lung, using curved multiplanar reconstruction. Using this software, we demonstrated in patients with COPD that FEV1 (\%predicted) was highly correlated with airway dimensions and the correlation coefficients improved as the airway became smaller in size (3). In this study, our aims are to further confirm the significant relationship between airway dimensions and airflow limitation in larger number of subjects, and to examine the relationship of airway dimensions with lung volumes in 95 patients with COPD (stage 0, 10; stage I, 23; stage II, 35; stage III, 24; stage IV, 3). We analyzed the airway dimensions from the $3^{\text {rd }}$ to the $6^{\text {th }}$ generations of the apical bronchus (B1) of the right upper lobe and the anterior basal bronchus (B8) of the right lower lobe. Lung volumes were measured by the helium closed circuit method. Both airway luminal area (Ai) and wall area percent (WA\%) of all the generations, except a few, from the two bronchi were significantly correlated with RV and RV/TLC, but not with TLC or FRC. More importantly, the correlation coefficients (r) between airway dimensions and RV/TLC improved as the airways became smaller in size from the $3^{\text {rd }}$ to $6^{\text {th }}$ generations in both

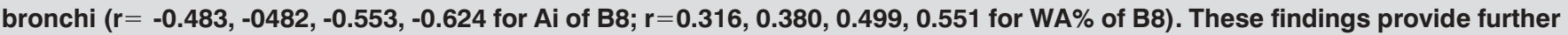
evidence that distal (small) airways rather than proximal (large) airways are the determinants for airflow limitation in COPD.

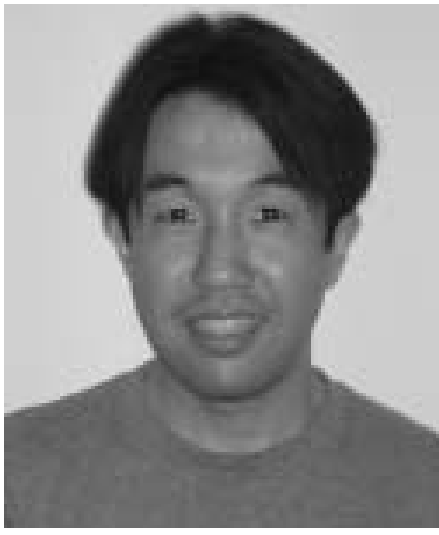

Masaru Hasegawa

Division of Respiratory Medicine, Dept of Internal Medicine, Hokkaido University Graduate School of Medicine, Sapporo, Japan

\section{MY JOB AND THE UNIT IN WHICH I WORK}

After graduating from Hokkaido University School of Medicine (Sapporo, Japan) in 1999, I started my professional career at Hokkaido University Hospital (Sapporo). After 2 yrs' training there, I gained further professional experience as a physician for another 4 yrs at several affiliated hospitals, which gave me valuable opportunities to see a variety of patients in internal medicine. During that period, I became interested in respiratory medicine because respiratory diseases are mysterious to me. In 2005, I decided to become a student at the Division of Respiratory Medicine, Hokkaido University Graduate School of Medicine (Hokkaido, Japan), where I wished to immerse myself in science and do some research in the field of respiratory medicine. Among four research groups in our division, I chose one headed by Prof. Nishimura, Assistant Prof. Betsuyaku and Assistant Prof. Nasuhara. The main theme of this research group is lung injury and repair and the target diseases include chronic obstructive pulmonary disease (COPD), interstitial pneumonia and acute lung injury. Their research covers all aspects, from bench to bedside, and I am particularly interested in the pathophysiology of COPD.

In 2003, our research group started the Hokkaido COPD cohort study, which was primarily designed to examine the natural history of COPD according to the phenotype based upon highresolution computed tomography (HRCT) findings and/or the reversibility of airflow limitation. They recruited almost 300 subjects with physician-diagnosed COPD at Hokkaido University Hospital and nine affiliated hospitals from May 2003 through to April 2005. At the first visit, we confirmed the diagnosis based on the spirometric criteria of the Global Initiative for Chronic Obstructive Lung Disease (GOLD) guideline. Moreover, for all the subjects, we conducted a detailed interview of disease history and symptoms, qualityof-life (QOL) measurement, blood sampling, pulmonary function tests and HRCT, which is a proven method for quantitative assessment of emphysema. Parallel to the cohort study, they wished to use computed tomography (CT) imaging to analyse airway disease in COPD. When I joined this research group, I was advised to be involved in this exciting research. Since then, I have been working energetically to develop a novel method capable of three-dimensional airway analysis, described later.

\section{MY WINNING POSTER AS PART OF MY RESEARCH}

Recent progress in CT technology has made it possible to detect and quantify airway abnormalities, and there have been only a few studies that have focused on measuring the airway dimensions in patients with COPD $[1,2]$. In previous reports, 

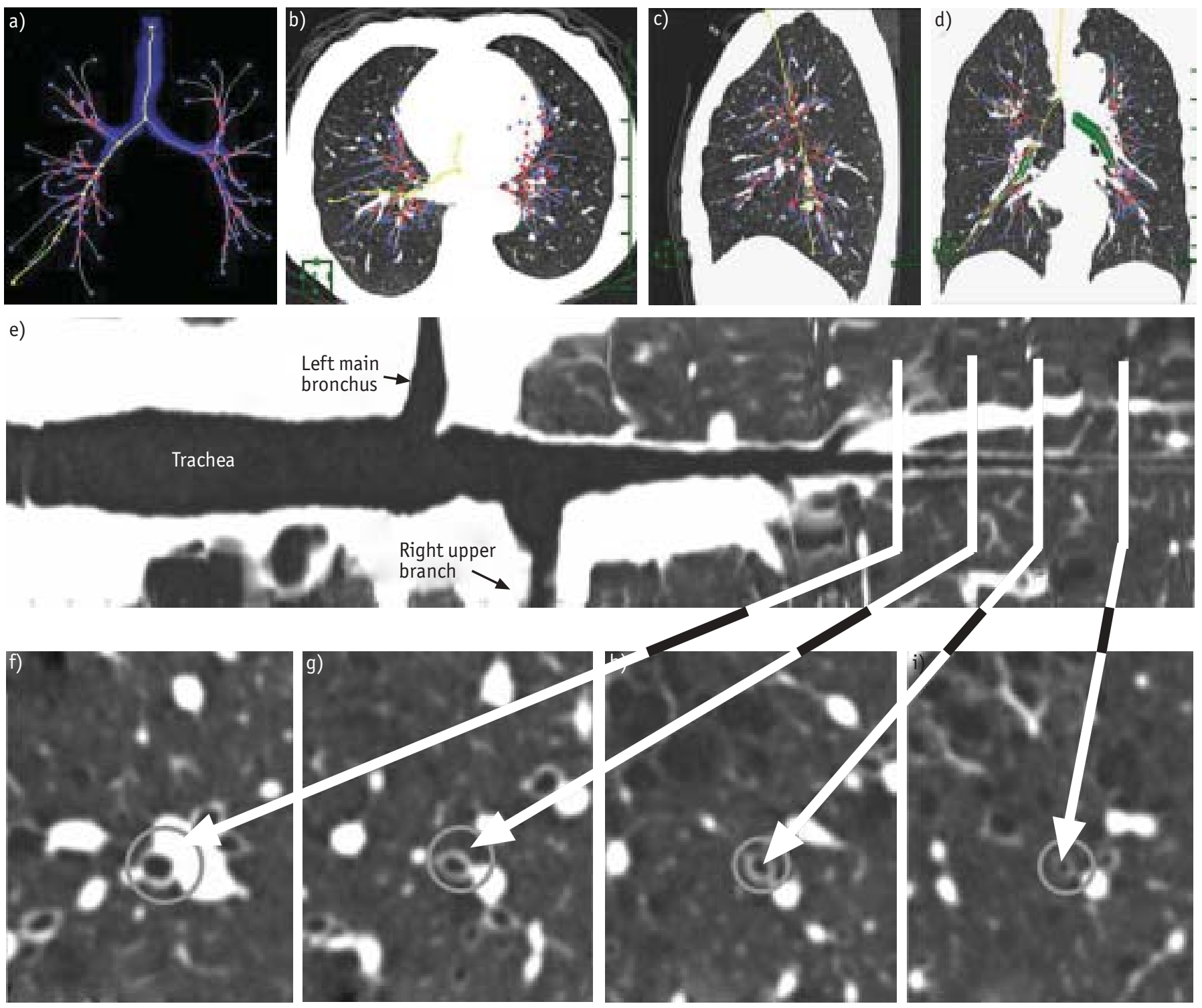

FIGURE 1. a) Accurate bronchial skeleton. Source images of b) axial, c) sagittal and d) coronal slices. Yellow lines indicate the same series of airway in parts a, b, $c$ and d. e) A curved multiplanar reconstruction (MPR) image of the selected airway from the right lower lobe. $\mathrm{f}, \mathrm{g}, \mathrm{h}$ and i) Short axis images obtained from the curved MPR image are precisely perpendicular to the long axis of the airway. The numbers of the bronchial generation are f) third, g) fourth, h) fifth and i) sixth.

accurate cross-sectional images could not always be obtained using conventional two-dimensional images because airways run in various directions in the lung. Therefore, we developed new software for measuring airway dimensions using curved multiplanar reconstruction (MPR), with which we could obtain longitudinal images and accurately analyse short-axis images of small airways with inner diameters as small as $2 \mathrm{~mm}$ located anywhere in the lung. Reconstructed data were transferred to the workstation and then reconstructed into three-dimensional chest images (AZE Ltd, Tokyo, Japan). The selected bronchial pathway was automatically converted to a curved MPR image and its long axis image appeared as if it was a straight pathway (fig. 1e). It is noteworthy that we could obtain short axis images that were exactly perpendicular to the long axis at any site (fig. 1f-i). Using the software, we analysed the relationship between airflow limitation and airway dimensions up to the sixth generation of bronchi in the upper lobe and the lower lobe of patients with COPD. We found that airway dimensions in two bronchi were significantly correlated with airflow limitation in patients with COPD. More importantly, the correlation coefficients ( $r$ ) improved as the airway became smaller in size from the third (segmental) to the sixth generations [3].

\section{MY RESEARCH AS PART OF MY WORKING GROUP/ RESEARCH TEAM}

The results shown in my winning poster fit well into the main research area of the Hokkaido COPD cohort study group led by Prof. Nishimura, who is also a director of the First Dept of Medicine at the Hokkaido University Hospital. The Hokkaido COPD cohort study is currently ongoing and, therefore, the analysis of airway disease will be applied in the longitudinal 
study; for example, the assessment of yearly changes or effects of medical intervention, etc. Furthermore, in our department, we study various pulmonary diseases, including bronchial asthma, pulmonary fibrosis and bronchiolits. The analysis of airway disease will be expanded to these diseases.

\section{THE IMPACT OF MY WORK ON CLINICAL OR RESEARCH PRACTICE}

In conclusion, we have demonstrated for the first time in in vivo in patients with COPD, that the forced expiratory volume in one second (\% predicted) and the residual volume/total lung capacity ratio was highly correlated with airway luminal area and, to a lesser extent, with wall thickening from the third to the sixth generation of both apical bronchus (B1) and basal bronchus (B8), and that the correlation coefficients improved as airway size decreased. These findings support the hypothesis that smaller airways, rather than larger airways, contribute more significantly to the airflow limitation in COPD. In the near future, with further advances in CT technology and in software, this type of approach will become even more attractive. Using this readily accessible and relatively noninvasive technique, we plan to conduct a longitudinal study of the subjects recruited for the Hokkaido COPD cohort study. Furthermore, using our technology and software, it might be possible to detect the effects of short-term and/or long-term pharmacological interventions on airway diseases in bronchial asthma as well as COPD.

\section{REFERENCES}

1 Nakano Y, Muro S, Sakai H, et al. Computed tomographic measurements of airway dimensions and emphysema in smokers: correlation with lung function. Am J Respir Crit Care Med 2000; 162: 1102-1108.

2 Orlandi I, Moroni C, Camiciottoli G, et al. Chronic obstructive pulmonary disease: thin-section CT measurement of airway wall thickness and lung attenuation. Radiology 2005; 234: 604-610.

3 Hasegawa M, Nasuhara Y, Onodera Y, et al. Airflow limitation and airway dimensions in chronic obstructive pulmonary disease. Am J Respir Crit Care Med 2006; 173: 1309-1315. 